\title{
Dielectric Characterization of Materials at Microwave Frequency Range
}

\author{
J. de los Santos*, D. Garcia, J.A. Eiras \\ Grupo de Cerâmicas Ferroelétricas - Departamento de Física \\ Universidade Federal de São Carlos \\ Rodovia Washington Luiz, km 235, 13565-905 São Carlos, SP - Brazil
}

Received: June 6, 2002; Revised: October 8, 2002

\begin{abstract}
In this study a coaxial line was used to connect a microwave-frequency Network Analyzer and a base moving sample holder for dielectric characterization of ferroelectric materials in the microwave range. The main innovation of the technique is the introduction of a special sample holder that eliminates the air gap effect by pressing sample using a fine pressure system control. The device was preliminary tested with alumina $\left(\mathrm{Al}_{2} \mathrm{O}_{3}\right)$ ceramics and validated up to $2 \mathrm{GHz}$. Dielectric measurements of lanthanum and manganese modified lead titanate (PLTM) ceramics were carried out in order to evaluate the technique for a high permittivity material in the microwave range. Results showed that such method is very useful for materials with high dielectric permittivities, which is generally a limiting factor of other techniques in the frequency range from $50 \mathrm{MHz}$ to $2 \mathrm{GHz}$.
\end{abstract}

Keywords: Dielectric characterization, microwave dispersion, ferroelectric ceramics

\section{Introduction}

Requirement of high-speed data transmission has led to a rising interest in the gigahertz region and has prompted research for dielectric resonators at microwave frequencies ${ }^{1}$. This is particularly true in the case of phase shifters used to electronically command steerable phase array antennas, and where large phase variations for producing radar scans are necessary. Thus, the use of ferroelectric materials in phase shifters has increased the investigation of these materials in the microwave range ${ }^{2}$ because its excellent ferroelectric and piezoelectric properties. Physical and dielectric properties such as relaxational and motional resonant mechanism can be investigated by analyzing the complex dielectric permittivity in a broad spectral frequency range (dielectric dispersion). For the study of orientational and relaxational polarization effects, automated measuring equipment, where some techniques using frequency response analysis (mainly by resonant cavity method) are usually applied and commercially available today.

The nature of the high frequency dielectric dispersion of ferroelectric ceramics in the range of $10^{6}-10^{9} \mathrm{~Hz}$ has been studied since $1940^{3,4}$. Dielectric dispersion spectra pro- vide the frequency regions where materials are useful for practical application ${ }^{5}$. Generally, at high frequencies the dielectric permittivity of ferroelectric materials is relatively low, facilitating their integration in microwave devices ${ }^{6}$. Microwave dielectric studies have been performed in a wide temperature range $(300 \mathrm{~K}-900 \mathrm{~K})^{7}$ mainly by coaxial technique using the reflectometric method $^{8}$. Reflection coefficients $\left(\Gamma^{\prime}, \Gamma^{\prime \prime}\right.$ : real and imaginary component, respectively) versus frequency are measured and the complex permittivity $(\varepsilon=\varepsilon '-\mathrm{j} \varepsilon$ ") $)$ is obtained. Simple configuration geometry of the sample combined with a wide frequency range and temperature interval ${ }^{9}$ are the most important advantages of this method. However, for high permittivity $\left(\varepsilon^{\prime}{ }_{r}>100\right)$, where $\varepsilon_{\mathrm{r}}^{\prime}=\varepsilon^{\prime} / \varepsilon_{\mathrm{o}}$ is the relative dielectric permittivity, and high dielectric loss materials real and imaginary parts of the reflection coefficient become difficult to be measured ${ }^{10}$. The dielectric response is governed mainly by resonance effects of the system when continuous frequency methods are used, which lead to camouflage the real dielectric behavior of the sample under test. To the best of our knowledge, few works focusing continuous frequency method can be found in the literature ${ }^{8}$. The goal of this work is to provide an alternative technique for dielectric dispersion measurements in a mi-

*e-mail: santos@df.ufscar.br 
crowave range of high permittivity materials by adapting a special sample holder at the end of a coaxial line. Using this system the real and imaginary permittivities of lead titanate based ferroelectric ceramics were measured.

\section{Experimental}

Dielectric characterization was made using a Network Analyzer HP-8719C that is responsible for the generation and reception of a signal in the frequency range of $50 \mathrm{MHz}$ to $2 \mathrm{GHz}$. Technical reason associated to our system configuration limited the accurated measurements to the frequency range of $50 \mathrm{MHz}-2 \mathrm{GHz}$, although Network Analyzer HP-8719C can generate signal up to $13 \mathrm{GHz}$. Equipment setup are in progress to higher frequency measurements. In order to obtain the dielectric response of materials the reflectometric method was employed. The setup known by coaxial probe method is represented in the fig. 1a. The sample is kept at rest on the end terminal of the probe. However in the case of very high dielectric permittivity $\left(\varepsilon^{\prime}{ }_{r}>100\right)$ this method presents some disadvantages: a) with increasing frequency the modulus of the reflection coefficient $|\Gamma|$ becomes close to 1 and its phase $\phi$ close to zero. Therefore they are difficult to be measured and the quality of the results largely depends on the initial calibration of the analyzer; b) air gaps formed between the sample and the inner/ outer conductors cause very large measurement errors when the sample is badly tooled and c- in high temperature range different thermal expansions of the sample and inner/outer conductors also can bring errors generated by the air gaps formation.
For reducing these limitations and successful characterize high dielectric permittivity materials an alternative coaxial line method was mounted. In this work a moving sample holder was adapted at the end of the coaxial line as shown in the Fig. 1b. The sample (4) is located at the center of the mobile base (8) of the sample holder (5) and coupled to the coaxial line (1) by threading the external fixer of the coaxial line (2) with the upper part (threaded) (9) of the sample holder. Adjusting the external fixer, the mobile base of the sample holder starts to move down while the sample holder is moving up. Thus, by adjusting the pressure, a better control of the sample position can be achieved in the coaxial line method. The pressure level is qualitatively controlled by using an adjusting pressure punch (6) coupled at the end of the sample holder. When the external fixer device is manipulated, the sample holder begins to turn along with the upper part of the adjusting pressure punch. The needle (7), fixed to the upper part of the adjusting pressure punch by an internal spiral spring, indicates the different positions corresponding to the different levels of pressure. A ring shaped metallic washer (3) is used to guarantee the inferior contact of the sample.

For determining the accurated dependence of the reflection coefficient parameters on the frequency, careful compensation procedure was carried out to account for spurious reflections that may result by transmission line discontinuities and also to eliminate the effect of the resistances and capacitances of the sample holder. Three different HP standard terminations (open, short and $50 \Omega$ ),
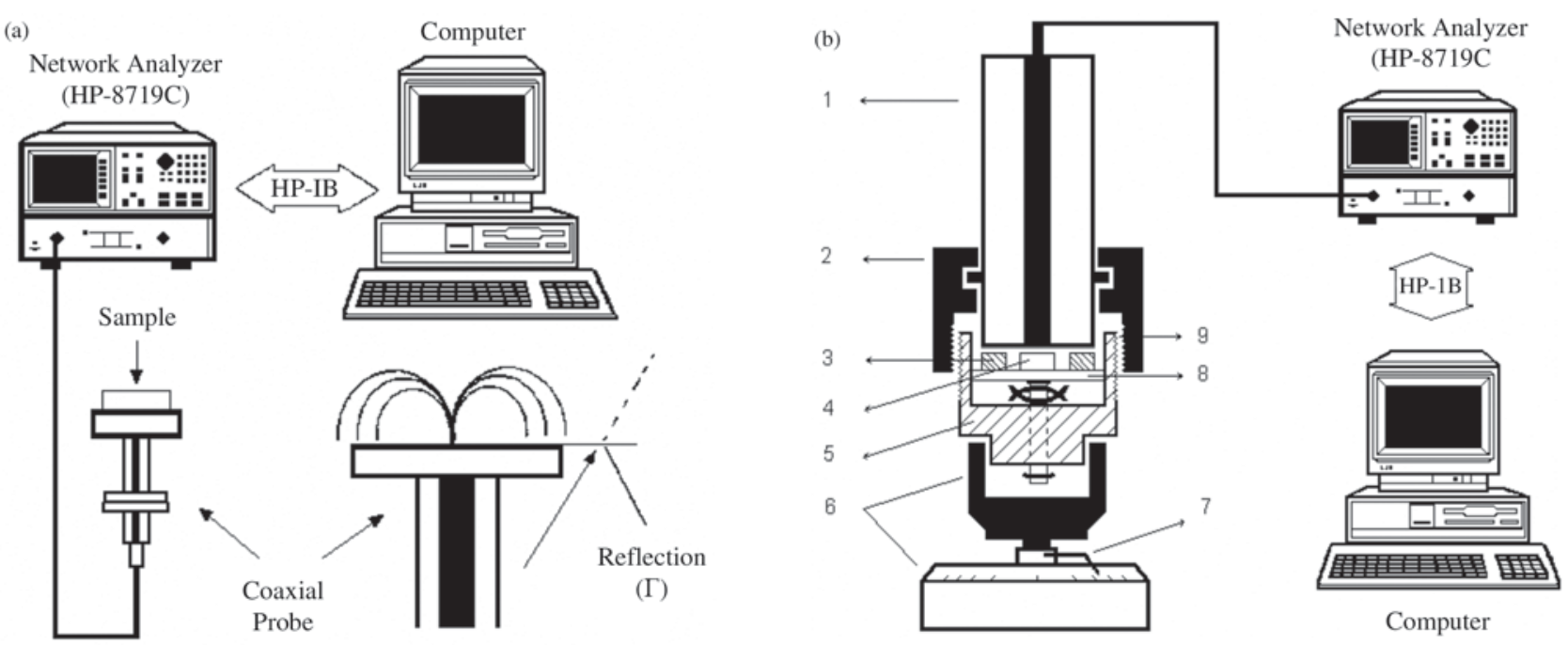

Figure 1. Experimental diagram for dielectric characterization using the reflectometric technique: (a), coaxial probe method and (b), coaxial line method with an adapted moving sample holder. 
with reflection coefficients of $1,-1$ and 0 , respectively, were used in the measured frequency range. Therefore, reflection coefficients $\Gamma^{\prime}$ and $\Gamma^{\prime \prime}$ data, corrected for the line effects, were frequency scanned and automatically transferred from the network analyzer to the computer. The real and imaginary parts of the complex dielectric permittivity were determined by using the following relations ${ }^{11}$.

$$
\begin{aligned}
& \mathcal{E}_{r}^{\prime}=\frac{1}{A f}\left[\frac{-2 \Gamma^{\prime \prime}}{\left(1+\Gamma^{\prime}\right)^{2}+\Gamma^{\prime \prime 2}}\right] \\
& \mathcal{E}^{\prime \prime}{ }_{r}=\frac{1}{A f}\left[\frac{1-\Gamma^{\prime 2}-\Gamma^{\prime \prime 2}}{\left(1+\Gamma^{\prime}\right)^{2}+\Gamma^{\prime \prime 2}}\right]
\end{aligned}
$$

where $f$ is the measuring frequency. A is determined by the characteristic impedance of the analyzer $\left(Z_{o}=50 \Omega\right)$ and the dimensions of the sample: as follow $A=2 \varepsilon_{o} \frac{\pi^{2} r^{2}}{t} Z_{o}$, where $\varepsilon_{\mathrm{o}}$ is the dielectric permittivity of free space; $r$ is the radius and, $t$, the thickness of the sample.

A ferroelectric ceramic corresponding to the PLTM system (solid solution of lanthanum manganese modified lead titanate) and an $\mathrm{Al}_{2} \mathrm{O}_{3}$ ceramic, with $\varepsilon_{\mathrm{r}}^{\prime} \approx 240^{12}$ and $\varepsilon_{\mathrm{r}}^{\prime} \approx 8^{13}$, respectively, were used for the dielectric characterization in the range of $50 \mathrm{MHz}$ to $2 \mathrm{GHz}$ at room temperature. The $\mathrm{Al}_{2} \mathrm{O}_{3}$ ceramic was chosen as reference material for the measurements. Air gaps at the interfaces between the coaxial line and sample were carefully controlled by an optimal sample polishing process, in order to guarantee parallel and flat faces. Alumina powder of $1 \mu \mathrm{m}$ was used in this polishing process. Gold electrodes were deposited by sputtering on the faces of the discs with $2.0 \mathrm{~mm}$ in diameter and $0.5 \mathrm{~mm}$ in thickness to insure good electric contacts.

\section{Results}

\subsection{Low permittivity measurement tests}

Figure 2 shows the dielectric response of the alumina ceramic obtained at room temperature using the commercial coaxial probe method (Fig. 1a). Results show very irregular dependence of the relative dielectric permittivities (real and imaginary parts) with the frequency in the whole range probably due to the air gaps. The air gap effect can be reduced by using the system of the Fig. $1 \mathrm{~b}$ (coaxial line), as described in the experimental procedure. As shown in Fig. 3, the obtained data were characterized by high stability without resonant effects in the whole frequency interval. Also, real part of the relative dielectric permittivity was 8.5 that is in agreement with data reported previously ${ }^{14-16}$. These results confirmed that the system and calibration procedure are suitable for microwave dielectric characterization.

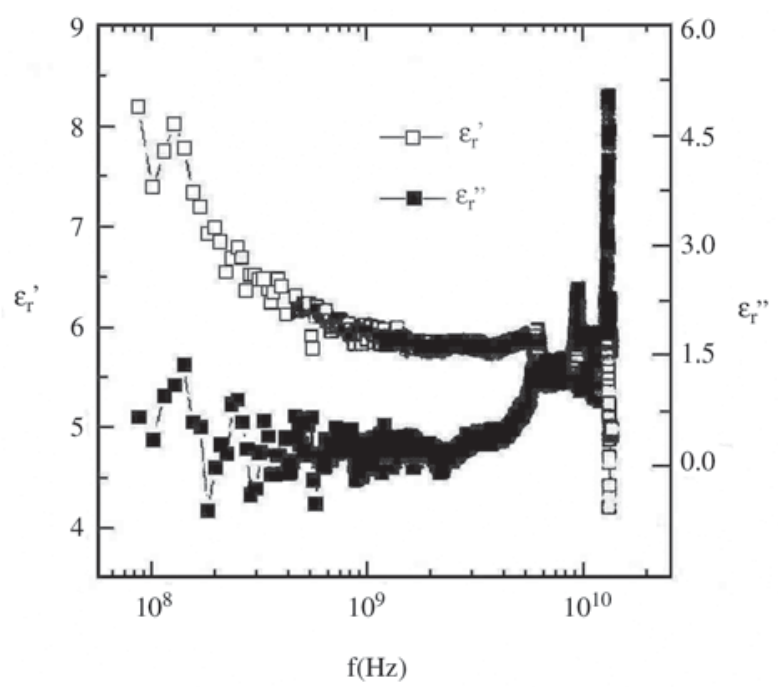

Figure 2. Dielectric response for the alumina ceramic by using the coaxial probe method, at room temperature.

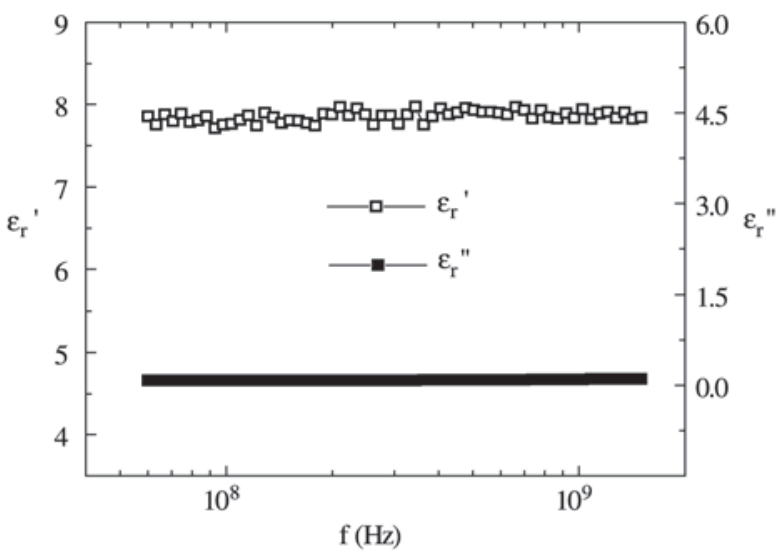

Figure 3. Dielectric response for the alumina ceramic by using the coaxial line method with the adapted moving sample holder, at room temperature.

\subsection{Dielectric measurements of a ferroelectric ceramic}

In order to evaluate our technique for high permittivity material measurements in the microwave range dielectric measurements were carried out in the PLTM ferroelectric ceramic. As followed for the $\mathrm{Al}_{2} \mathrm{O}_{3}$ ceramic calibration procedures were performed with different pressure levels on the sample. Figures $4 \mathrm{a}$ and $4 \mathrm{~b}$ show curves corresponding to two different pressures, $\mathrm{Pa}$ and $\mathrm{Pb}(\mathrm{Pa}>\mathrm{Pb})$. Since low lanthanum content lead titanate samples are characterized by an absence of dielectric relaxation phenomenon ${ }^{17}$ (frequency independent dielectric permittivity), for a temperature far away from the transition temperature, the obtained 
(a)

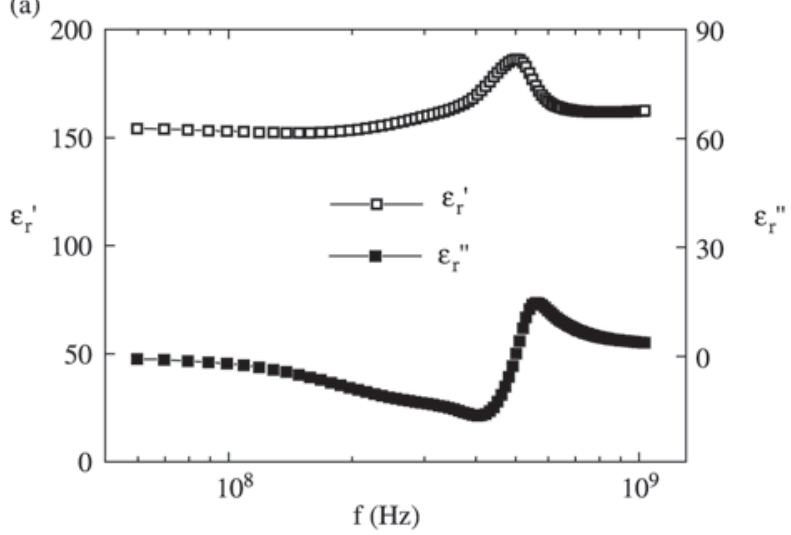

(b)

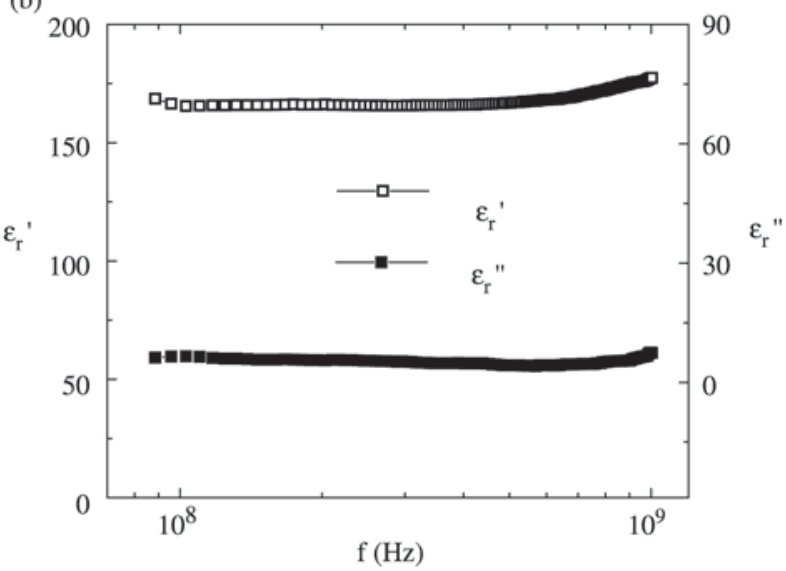

Figure 4. Dielectric response of the PLTM ferroelectric ceramic for two pressure levels on sample: $\mathrm{Pa}>\mathrm{Pb} . \mathrm{Pb}$ is in the limit of the minimum pressure necessary for the elimination of resonant effects.

curve in Fig. 4b seems to better represent the PLTM dielectric response. This result confirm that there exist a minimum pressure on the sample for which the resonant effects of the system disappear and the dielectric measurements are just characterized by the dielectric response of the sample, without any additional effects that might camouflage the dielectric measurements.

For comparison, low frequency dielectric data measured by using an HP-4194A Impedance/Gain-Phase Analyzer $(100 \mathrm{~Hz}-10 \mathrm{MHz}$ ) have been added to microwave results as shown in Fig. 5. The smooth continuity in the values of real and imaginary parts of dielectric permittivity for low and high frequency confirms the accuracy of the results obtained at the microwave frequency range. Also, $\varepsilon^{\prime}$ and $\varepsilon^{\prime \prime}{ }_{r}$ values are in agreement with those found in Ref. 12. It is important to point out that an optimal control of the pressure adjustment should be guaranteed, specifically for relaxor ferroelectrics materials, because of the presented relaxation

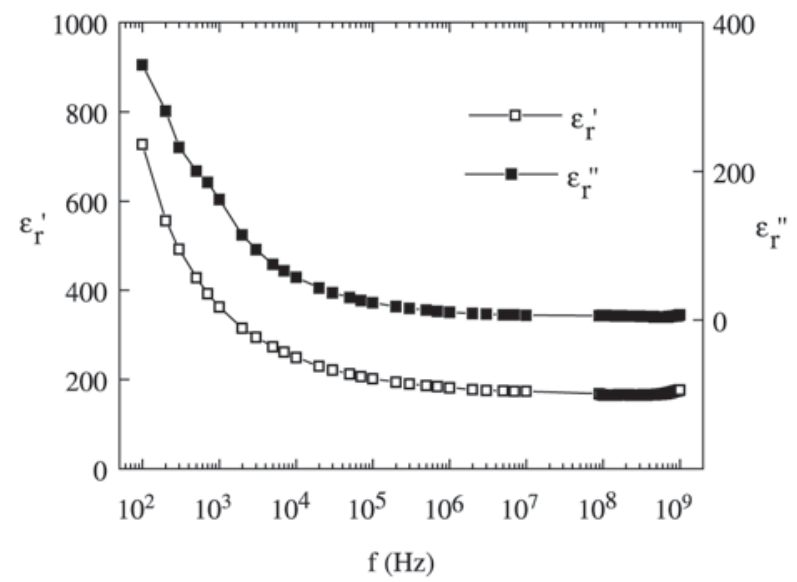

Figure 5. Dielectric response for the PLTM ferroelectric ceramic including the low and high frequency range $(100 \mathrm{~Hz}$ to $2 \mathrm{GHz})$, at room temperature.

behavior. Piezoelectric resonance effects of the material may mask this dielectric relaxation behavior when high pressure levels are used. These resonant effects arise from the piezoelectric $^{18}$ phenomenon showed by the ferroelectric materials when a mechanical strain is applied by static pressure.

\section{Conclusions}

Preliminary results on microwave dielectric characterization using an adaptation of the sample holder in the reflectometric method, demonstrates that the technique can be very useful for the study of materials with relative dielectric permittivities higher than 100 . Using this new system, characterized by a simple configuration geometry and the very small size of the sample is possible to obtain the temperature dependence of dielectric response in a wide range of microwave frequency, which was not possible at the moment by using the commercial coaxial probe method.

\section{Acknowledgements}

The authors thank to CAPES, CNPq and FAPESP Brazilian agencies for financial support. The authors also thank Dr. Manuel H. Lente (DF-UFSCar) for his valuable comments and Mr. Francisco J. Picon (DF-UFSCar) for technical assistance.

\section{References}

1. Babbitt, R.; Koscica, T.; Drach, W.; Didomenico. Integrated Ferroelectrics, v. 8, p. 65-76, 1995.

2. Zimmermang, F.; Voigts, M.; Weil, C.; Jakoby, R.; Wang, P.; Menesklou, W.; Ivers-Tiffée, E. J. Eur. Ceram. Soc., v. 21, p. 2019-2023, 2001. 
3. Hippel, A.V. Dielectric Material and Applications, John Wiley \& Sons, Inc.: New York; Chapman \& Hall, LTD.: London, p. 3-40, 1954.

4. Zcheludev, I.S. Physics of Crystalline Dielectrics. Electrical Properties, v.2. Plenum Press: New York-London, p. 455-531, 1971.

5. Xu, Y. Ferroelectric Materials and their Applications, Elsevier Science Publisher B.V., North-Holland, Amsterdam, p. 164-193, 1991.

6.Grigas, J. Microwave Dielectric Spectroscopy of Ferroelectrics and Related Materials. Ferroelectricity and Related Phenomena, Gordon and Breach Sci. Publish., v. 9, p. 9-108, 1996.

7. Mouhsen, A.; Achour, M.E.; Miane, J.L.; Ravez, J. Eur. Phys. J.Appl. Phys. v. 15, n. 2, p. 97-104, 2001.

8. Böhmer, R.; Maglione, M.; Lunkenheimer, P.; Loidl A. J. Appl. Phys., v. 65, n. 3, p. 901-904, 1989.

9. Bassora, L.A.; Eiras, J.A. Ferroelectrics, v. 223, n.1-4, p. 285-292, 1999.
10. Maglione, M.; Böhmer, R.; Loidl, A.; Höchli, U.T. Phys. Rev. B, v. 40, n. 16, p. 11441-11444, 1989.

11. Bassora, L. A. Caracterização dielétrica de materiais ferroelétricos na região de microondas, $\mathrm{Ph}$. D. Thesis, Federal University of São Carlos, São Carlos-SP, 1999.

12. García, D.; Eiras, J.A. Ferroelectrics, v. 123, p. 51-59, 1991.

13.Kim, J.C.; Schilling C. H.; Tomasik, P.; Auh, K.H. Mat. Lett., v. 42, p. 221-224, 2000.

14. Heintz, J.M.; Letullier, P.; Miane, J.L.; Ravez, J. Mat. Sci. Eng. B, v. 52, p. 84-88, 1998.

15. Ravinder, D.; Latha, K. Mat. Lett., v. 41, p. 247-253, 1999.

16. Haijun, Z.; Xi, Y.; Liangying, Z. Mat. Sci. Eng. B, v. 84, p. 252-257, 2001

17. Jonscher, A.K. J. Phys. D: Appl. Phys., v. 32, p. 57-70, 1999.

18. Jaffe, B.; Cook, W.R.; Jaffe, H. Piezoelectric Ceramics, Academic Press, London-New York, p. 7-279, 1971. 